\title{
SUBGRID-SCALE MODEL BASED ON THE INVARIANTS OF THE GRADIENT MODEL TENSOR
}

\author{
D.FOLCH ${ }^{1}$, F.X.TRIAS ${ }^{1}$, A.GOROBETS ${ }^{2}$, A.OLIVA $^{1}$ \\ ${ }^{1}$ Heat and Mass Transfer Technological Center, Technical University of Catalonia \\ C/Colom 11, 08222 Terrassa (Barcelona) \\ davidf@cttc.upc.edu,xavi@cttc.upc.edu, oliva@cttc.upc.edu http://www.cttc.upc.edu/ \\ ${ }^{2}$ Keldysh Institute of Applied Mathematics \\ 4A, Miusskaya Sq., Moscow 125047, Russia \\ andrey@cttc.upc.edu https://keldysh.ru
}

Key words: LES, SGS models, gradient model, turbulence

\begin{abstract}
The incompressible Navier-Stokes equations stand as the best mathematical model for turbulent flows. However, direct numerical simulations at high Reynolds numbers are not yet feasible because the convective term produces far too many relevant scales of motion, thus remaining limited to relatively low-Reynolds numbers. Dynamically less complex mathematical formulations have been developed for coarse-grain simulations, like the well known eddy-viscosity models. Most of these models are based on the combination of invariants of a symmetric tensor that depends on the gradient of the resolved velocity field, $G=\nabla \bar{u}$, and should properly detect different flow configurations (laminar and 2D flows, near-wall behavior, transitional regime, etc.). Brand-new models have been constructed considering the first three invariants of the symmetric tensor $\mathrm{GG}^{T}$ with excellent results; ${ }^{1}$ hence, it is formally based on the lowest-order approximation of the subgrid stress tensor, $\tau(\bar{u})=\frac{\Delta^{2}}{12} \mathrm{GG}^{T}+O\left(\Delta^{4}\right)$, i.e. the gradient model proposed by Clark. ${ }^{2}$ Furthermore, these models have been implemented on a pseudo-spectral algorithm with a fully-explicit second-order time-integration method. ${ }^{3}$ The performance of this special configuration has been successfully tested for decaying isotropic turbulence and a turbulent channel flow. It is currently being developed for a semi-infinite boundary layer with periodic conditions as a previous step to carry out wind farm simulations. Details of the implementation and numerical results will be presented.
\end{abstract}

\section{INTRODUCTION}

The incompressible Navier Stokes equations stand as the most useful tool to deal with the turbulent flows. However, for large Reynolds numbers, that is, when it exists many relevant scales of motion of the flow, the direct numerical simulation is unfeasible or a very high demanding resources procedure. There have been developed a lot of numerical methods to try to solve this issue. To achieve the highest efficiency, it is necessary to discriminate between them when dealing with specific problems. In our case, the final goal is to simulate a wind farm. As Menevau and Katz ${ }^{4}$ point out, most successful tests typically use spectral methods and cutoff filtering on homogeneous directions. Our approach, then, is that of a pseudo-spectral algorithm. The remaining work is to find the best numerical method compatible with boundary layers, 
spectral decomposition, and accurateness.

One that has given good results in many cases is that of the well known Large Eddy Simulation. Most of the models of the LES algorithms are based on combinations of invariants of some tensor depending on the gradient of the velocity. For example, they are the Smagorinsky model, ${ }^{5}$ Vreman's, ${ }^{6}$ WALE, ${ }^{7}$ or the recently deployed S3PQR models. ${ }^{1}$ To assure the validity of the models, usually they are tested on what is called benchmark cases and compared with experimental results. These cases are, for example, the channel flow, the sink flow, the homogeneous isotropic turbulence, the boundary layer, and so on.

Besides, there are some technical specifications of the algorithm that could yield different performances of the model. That is, for example, the time-stepping procedure, the characteristic of the domain, or the mathematical approximations.

In this article, we will show the performance of the S3PQR LES models on the free boundary layer case, with a pseudo-spectral, fractional step method configuration. Therefore, we will review the technical aspects of the algorithm in the first next sections. Finally, we will present the results and compare them through several other LES methods towards finding the best method for a wind farm simulation.

\section{PSEUDO-SPECTRAL S3PQR MODEL}

\subsection{Foundations of LES}

Large eddy simulation equations result from applying a spatial filter to the incompressible Navier-Stokes equations, that then read as follows:
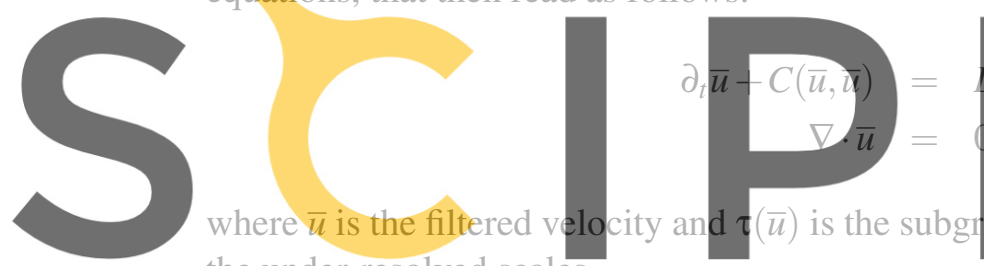

the under-resolved scales.
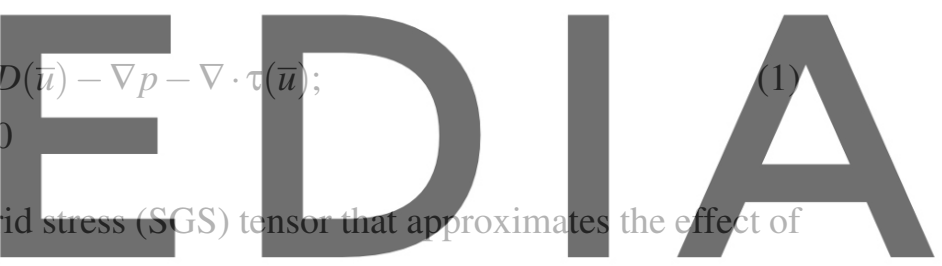

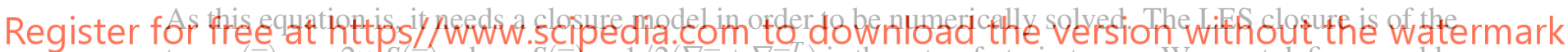
type $\tau(\bar{u}) \approx-2 v_{e} S(\bar{u})$ where $S(\bar{u})=1 / 2\left(\nabla \bar{u}+\nabla \bar{u}^{I}\right)$ is the rate-of-strain tensor. We must define an eddy viscosity: $v_{e}=\left(C_{m} \Delta\right)^{2} D_{m}(\bar{u})$ where $C_{m}$ is the model constant, $\Delta$ is the subgrid characteristic length, and $D_{m}(\bar{u})$ is the differential operator with units of frequency associated with the model ${ }^{8}$

\subsection{The velocity gradient tensor}

From the several mathematical invariants that can be calculated from the gradient tensor $G=\nabla \bar{u}$, we will restrict ourselves on

$$
\left\{Q_{\mathrm{G}}, R_{\mathrm{G}}, Q_{\mathrm{S}}, R_{\mathrm{S}}, V_{\mathrm{G}}^{2}\right\}
$$

where, given a second-order tensor A, one can define ${ }^{1}$

$$
\begin{aligned}
Q_{A} & =(1 / 2)\left(\operatorname{tr}^{2}(A)-\operatorname{tr}\left(A^{2}\right)\right) \\
R_{A} & =\operatorname{det}(A) \\
V_{\mathrm{G}}^{2} & =4\left(\operatorname{tr}\left(S^{2} \Omega^{2}\right)-2 Q_{S} Q_{\Omega}\right) \\
P_{A} & =\operatorname{tr}(A)
\end{aligned}
$$


where $S=1 / 2\left(G+G^{T}\right)$ and $\Omega=1 / 2\left(G-G^{T}\right)$ are the symmetric and the skew-symmetric parts of the gradient tensor, respectively.

Most of the LES known models are based upon a combination of these invariants ${ }^{8}$ and can be written as a funcion explicitly depending on them:

- Smagorinsky ${ }^{5}$ model $v_{e}^{\text {Smag }}=f\left(Q_{\mathrm{S}}\right)$

- Verstappen's ${ }^{9}$ model $v_{e}^{V e}=f\left(R_{\mathrm{S}}, Q_{\mathrm{S}}\right)$

- WALE $\operatorname{model}^{7} v_{e}^{W}=f\left(Q_{\mathrm{G}}, V, Q_{\mathrm{S}}\right)$

- Vreman's model ${ }^{6} v_{e}^{V r}=f\left(V, Q_{\mathrm{G}}, Q_{\Omega}, Q_{\mathrm{S}}\right)$

- $\sigma$-model ${ }^{8} v_{e}^{\sigma}=f\left(\mathrm{GG}^{T}\right.$ eigenvalues $)$

\subsection{S3PQR model foundations}

The recently developed S3PQR models ${ }^{1}$ involve three invariants of the symmetric tensor $\mathrm{GG}^{T}$ formally based on the lowest-order approximation of the subgrid stress tensor, ${ }^{2}$

$$
\tau(\bar{u})=\frac{\Delta^{2}}{12} \mathrm{GG}^{T}+O\left(\Delta^{4}\right)
$$

These invariants are directly related with the previous ones

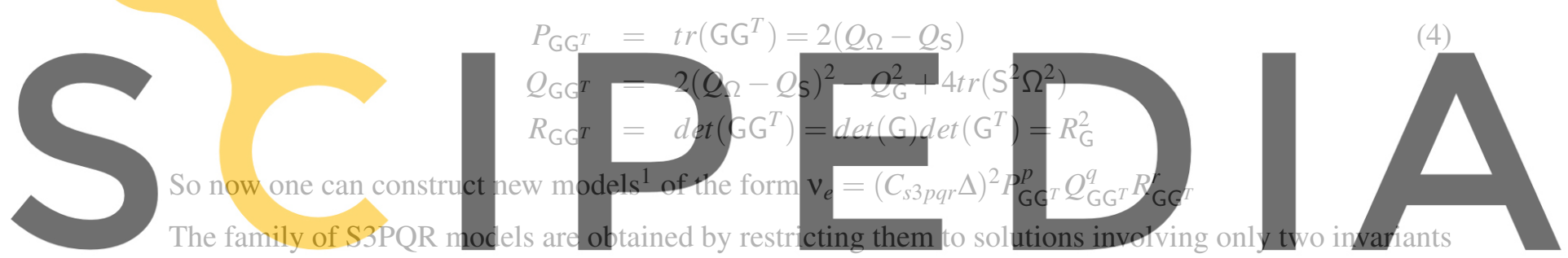

of $\mathrm{GG}^{T}$, so three different models can be found ${ }^{1}$

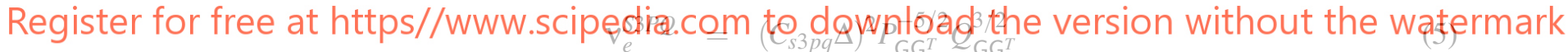

$$
\begin{aligned}
v_{e}^{S 3 P R} & =\left(C_{S 3 p r} \Delta\right)^{2} P_{\mathrm{GG}^{T}}^{-1} R_{\mathrm{GG}}^{1 / 2} \\
v_{e}^{S 3 Q R} & =\left(C_{s 3 q r} \Delta\right)^{2} Q_{\mathrm{GG}^{T}}^{-1} R_{\mathrm{GG}^{T}}^{5 / 6}
\end{aligned}
$$

\subsection{Some properties of S3PQR models}

For completeness, we will give here a brief review of some of the properties of the S3PQR. ${ }^{1,8}$ First of all, there remains to be determined the model constant and it was done in two ways:

One is imposing numerical stability and less or equal dissipation than Vreman's model $C_{s 3 p q}=C_{s 3 p r}=$ $C_{s 3 q r}=\sqrt{3} C_{V r} \approx 0.458$

The other one is granting than the averaged dissipation of the models is equal than that of the Smagorinsky model. $C_{s 3 p q}=0.572, C_{s 3 p r}=0.709, C_{s 3 q r}=0.762$

In some cases, there have been found differences in the numerical results depending on the constant chosen. For example, simulations of decaying isotropic turbulence have shown that only the last values provide the right SGS dissipation. ${ }^{1}$ 
Another important property of each of the S3PQR model is its $2 \mathrm{D}$ behaviour: ${ }^{1,8}$ only $R_{\mathrm{GG}^{T}}$-dependent models switch off for 2D flows, so S3PR and S3QR models are preferable in this case.

Other general properties are positiveness, locality, Galilean invariance, and proper near-wall behaviour ${ }^{10}$ $\left(O\left(y^{3}\right)\right.$ dependence on normal direction).

\subsection{Generalities about the pseudo-spectral configuration}

We are working through a pseudo-spectral method, ${ }^{3}$ with Fourier expansion in the streamwise and spanwise directions, and Chebyshev expansion for the normal one. It is implemented on the structured nonstaggered grid. It applies $3 / 2$ rule de-aliasing technique.

It is worth mentioning that we are using the usual strong Navier Stokes formulation, so we have to solve the Poisson equation for the pressure.

The temporal configuration is a fully-explicit second-order Adams-Bashforth time-integration method, and the computation code is based on MPI parallelization.

With these features, the models have been tested and validated for the homogeneous isotropic turbulence case,${ }^{1}$ both in the decaying and forced simulations, showing good results compared with classical ComteBellot \& Corrsin experiment, ${ }^{11}$ for example. The channel flow has been tested ${ }^{12}$ also and compared with the DNS of Moser et al., ${ }^{13}$ yielding again proof of validity.

\section{THE BOUNDARY LAYER}
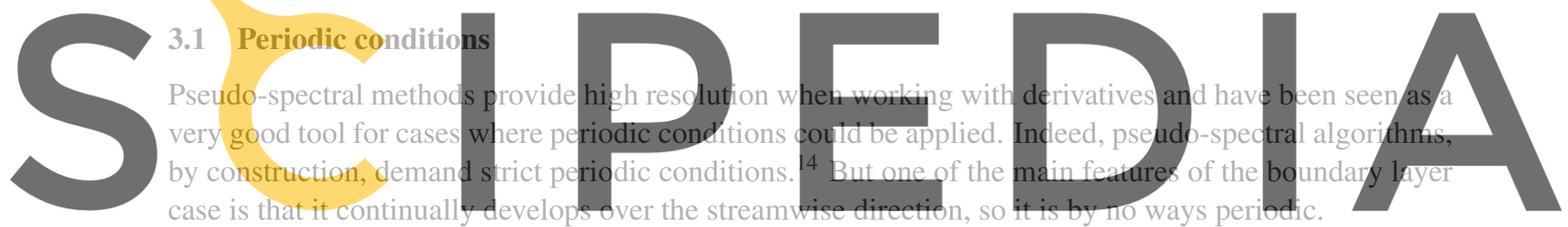

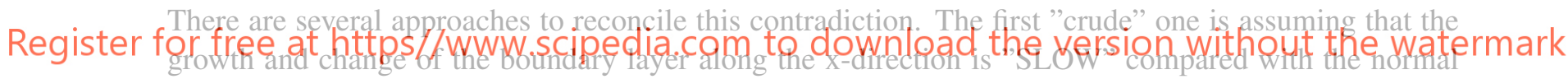
direction.

More elaborated is the method proposed by Spalart, ${ }^{15,16}$ which includes normal coordinate similarity transformations, growing terms $G T(\bar{u}, \bar{U})$ and several other assumptions. Moreover, Spalart developed two separate global methods, the first one with a single domain, ${ }^{15}$ and the other one, splitting the domain into several stations. ${ }^{16}$

Since our main objective is to check whether the S3PQR models can be adapted to the special characteristics of the boundary layer in a pseudo-spectral manner, we will not insist or discuss further the validity of the periodic configuration.

Therefore, we will follow the strategy of testing the algorithm from the crudest approach to the several stations model and compare its performance with other LES models. 


\subsection{Numerical technicalities of the boundary layer}

The general structure of the algorithm is a direct adaptation of the channel flow code, already successfully tested. ${ }^{1,3}$

It is based on the strong formulation of the Navier-Stokes equations with a Poisson - pressure correction term. The main changes are, as expected, on the initial velocity field, the boundary conditions, the algebraic scaling, and the Poisson solver.

The boundary conditions are the usually prescribed ones:

$$
\begin{aligned}
& y=0 \rightarrow u, v, w=0 \\
& y=\infty \rightarrow\langle u>=1 ; v, w=0
\end{aligned}
$$

We are dealing only with the zero mean pressure gradient case.

One of the key issues of the boundary layer simulations is the semi-infinite domain and the scaling procedure over the normal direction, from $y \varepsilon[0, \infty)$ to $y \varepsilon[-1,1]$, to deal with it.

The Navier-Stokes must be reformulated in presence of this scaling factor (sc):

$$
\begin{aligned}
\partial_{t} \bar{u}+C_{s c}(\bar{u}, \bar{u})+G T(\bar{u}, \bar{U}) & =D_{s c} \bar{u}-\nabla_{s c} p-\nabla_{s c} \cdot \tau(\bar{u}) ; \\
\nabla_{s c} \cdot \bar{u} & =0
\end{aligned}
$$

\subsection{Scaling procedure}

For this model we have used the
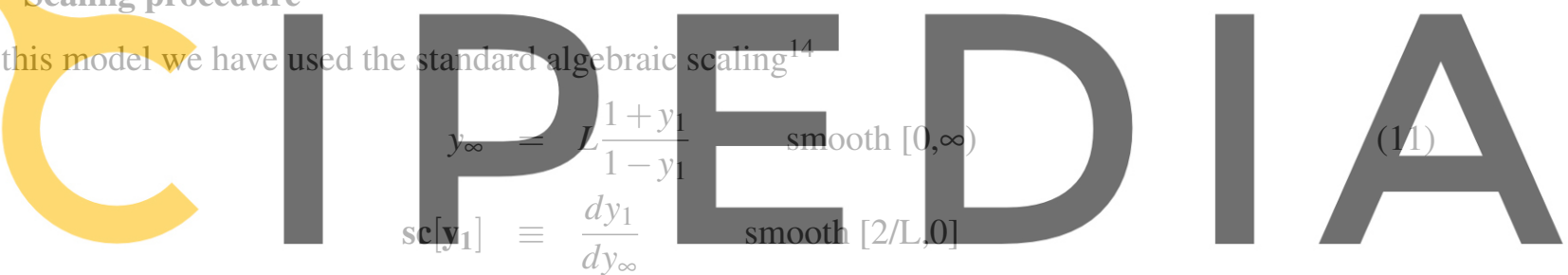

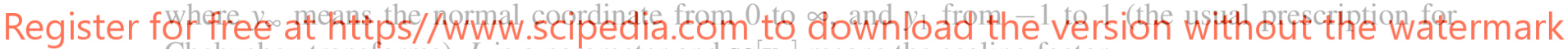 Chebyshev transforms), $L$ is a parameter and sc $\left[\mathbf{y}_{1}\right]$ means the scaling factor.}

The fixing of the $L$ parameter is somewhat arbitrary: the value is barely related to the number of points in the normal direction. ${ }^{14}$ But again, we will not enter into this discussion any further. We have fixed the value of $L=5$, since that is a reasonable election.

For clarity, we show the change that the scaling produces in some of the terms of the Navier-Stokes equations. So, for the $\mathrm{x}$-direction:

- The convective term:

$$
u \frac{\partial u}{\partial x}+v \cdot \mathbf{s c}\left[\mathbf{y}_{\mathbf{1}}\right] \frac{\partial u}{\partial y_{1}}+w \frac{\partial u}{\partial z}
$$

- The diffusive term:

$$
v\left(\frac{\partial^{2} u}{\partial x^{2}}+\mathbf{s c}\left[\mathbf{y}_{\mathbf{1}}\right] \frac{\partial}{\partial y_{1}}\left(\mathbf{s c}\left[\mathbf{y}_{\mathbf{1}}\right] \frac{\partial u}{\partial y_{1}}\right)+\frac{\partial^{2} u}{\partial z^{2}}\right)
$$

It is apparent that there are no special difficulties on both these terms. 
- The Poisson equation:

$$
\frac{\partial^{2} P}{\partial x^{2}}+\mathbf{s c}\left[\mathbf{y}_{1}\right] \frac{\partial}{\partial y_{1}}\left(\mathbf{s c}\left[\mathbf{y}_{1}\right] \frac{\partial P}{\partial y_{1}}\right)+\frac{\partial^{2} P}{\partial z^{2}}=\frac{\partial u}{\partial x}+\mathbf{s c}\left[\mathbf{y}_{\mathbf{1}}\right] \frac{\partial v}{\partial y_{1}}+\frac{\partial w}{\partial z}
$$

Now, the Poisson equation involves not only the second derivative of the pressure, but also the first. It changes all the structure of the matrix equation. Moreover, it introduces spurious terms that must be carefully removed to give the maximum accuracy possible. The solver then, is profoundly changed.

- The S3PQR algorithm

$$
\frac{\partial}{\partial x}\left(v_{e}[x, y, z] \frac{\partial u}{\partial x}\right)+\mathbf{s c}\left[\mathbf{y}_{1}\right] \frac{\partial}{\partial y_{1}}\left(v_{e}[x, y, z] \cdot \mathbf{s c}\left[\mathbf{y}_{1}\right] \frac{\partial u}{\partial y_{1}}\right)+\frac{\partial}{\partial z}\left(v_{e}[x, y, z] \frac{\partial u}{\partial z}\right)
$$

The scaling factor enters into the $v_{e}$ calculation via the $\Delta$ subgrid characteristic length and the several invariants involved.

They are the relevant changes introduced concerning channel flow code. Not all of them are minor changes: we must keep in mind that algebraic scaling introduces great numerical expansions. Let us see the results.

\section{RESULTS}

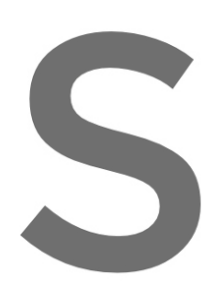

\subsection{Crude approximation}

For the crude approximation, th

any growing terms, there

Reynolds number, the diss no meaningful results.
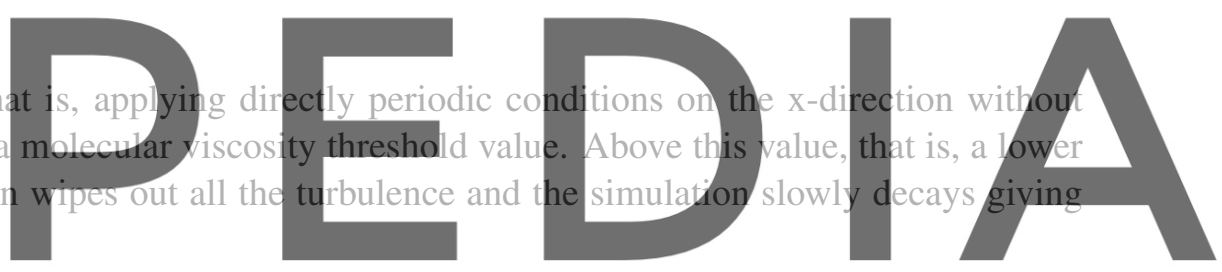

Below this viscosity value, the turbulence can be generated at the wall and maintained over the time,

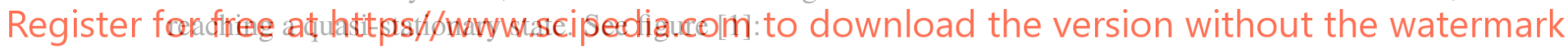

The net balance of the viscosity effect over the model is a tricky one. For models like Smagorinsky and Vreman's, the increased viscosity (via eddy viscosity) gives bad results. Also for WALE.

For the case of no model, we obtain some better results: it can reproduce the-law-of-the-wall velocity profile but it fails to follow the logarithmic-law away from the wall.

The results are far better with the S3PQR algorithms, but with some differences among them. It must be stretched that the numerical constant of the S3PQR models is of the Vreman's type $C_{s 3 p q}=C_{s 3 p r}=$ $C_{s 3 q r}=\sqrt{3} C_{V r}$. Using the other type (Smagorinsky) gives again bad results.

\subsection{Further research}

It is currently "on progress" the incorporation of Spalart's growing terms and their simulation on a single or several stations. As a final step, the code will be tested for a wind farm facility with mathematically simplified models of turbines. 


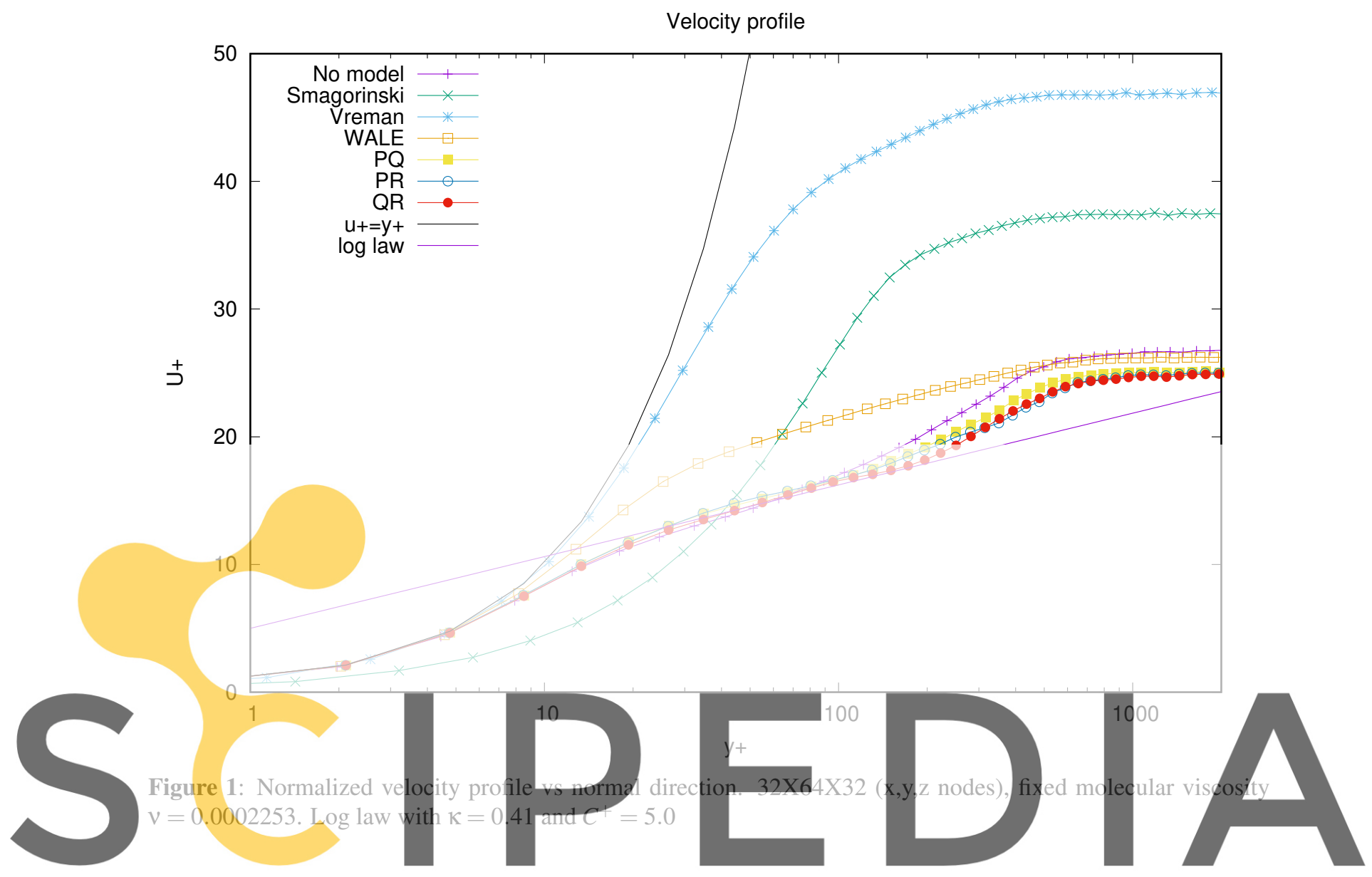

5 CONCLUSIONS

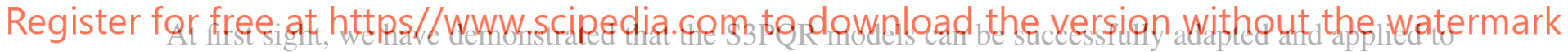

the constraining characteristics of a boundary layer. At least for the crude approximation (that is, direct periodic treatment of the $\mathrm{x}$-direction), they give better qualitative resuits than the no modei simulation and also than some of other LES algorithms. Therefore, they are possible candidates to perform practical simulations of wind farm facilities.

\section{ACKNOWLEDGEMENTS}

The work has been financially supported by a competitive R+D project (ENE2017-88697-R) by the Spanish Research Agency. The authors thankfully acknowledge these institutions.

\section{REFERENCES}

[1] F. X. Trias, D. Folch, A. Gorobets, and A. Oliva, "Building proper invariants for eddy-viscosity subgrid-scale models," Physics of Fluids, vol. 27, no. 6, p. 065103, 2015.

[2] R. A. Clark, J. H. Ferziger, and W. C. Reynolds, "Evaluation of subgrid-scale models using an accurately simulated turbulent flow," Journal Fluid Mechanics, vol. 91, pp. 1-16, 1979. 
[3] F. X. Trias, D. Folch, A. Gorobets, and A. Oliva, "Spectrally-consistent regularization of NavierStokes equations," Journal of Scientific Computing, vol. 79, pp. 992-1014, 2019.

[4] C. Meneveau and J. Katz, "Scale-Invariance and Turbulence Models for Large-Eddy Simulation," Annual Review of Fluid Mechanics, vol. 32(1), p. 1-32, 2000.

[5] J. Smagorinsky, "General Circulation Experiments with the Primitive Equations," Monthly Weather Review, vol. 91, pp. 99-164, 1963.

[6] A. W. Vreman, "An eddy-viscosity subgrid-scale model for turbulent shear flow: Algebraic theory and applications," Physics of Fluids, vol. 16, no. 10, pp. 3670-3681, 2004.

[7] F. Nicoud and F. Ducros, "Subgrid-scale stress modelling based on the square of the velocity gradient tensor," Flow, Turbulence and Combustion, vol. 62, no. 3, pp. 183-200, 1999.

[8] F. Nicoud, H. B. Toda, O. Cabrit, S. Bose, and J. Lee, "Using singular values to build a subgrid-scale model for large eddy simulations," Physics of Fluids, vol. 23, no. 8, p. 085106, 2011.

[9] R. Verstappen, "When does eddy viscosity damp subfilter scales sufficiently?," Journal of Scientific Computing, vol. 49, no. 1, pp. 94-110, 2011.

[10] D. R. Chapman and G. D. Kuhn, "The limiting behaviour of turbulence near a wall," Journal of Fluid Mechanics, vol. 170, pp. 265-292, 1986.

[11] G. Comte-Bellot and S. Corrsin, "Simple Eulerian time correlation of full- and narrow-band velocity signals in grid-generated, isotropic turbulence," Journal of Fluid Mechanics, vol. 48, pp. 273-

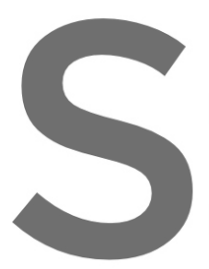
337,1971

[12] F. X. Trias, A. Gorobets, a spatially varying (edcy-) vis

[13] R. D. Moser, J. Kim. and up to $R e_{\tau}=590$,
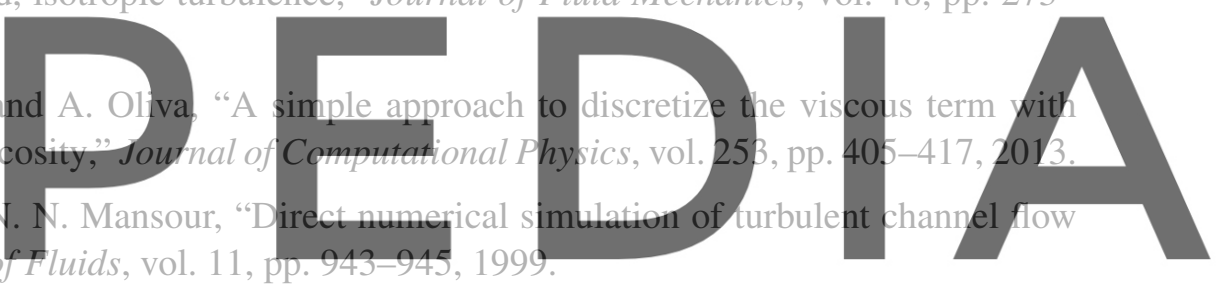

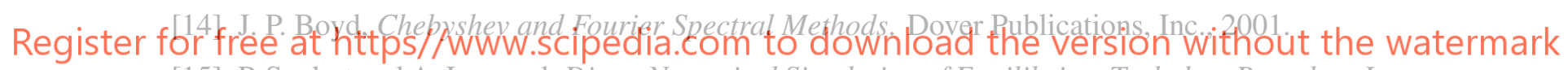

[15] P. Spalart and A. Leonard, Direct Numerical Simulation of Equilibrium Turbulent Boundary Layers. Berlin: Springer-Verlag, 1987.

[16] P. Spalart, "Direct simulation of a turbulent boundary layer up to $R_{\theta}=1410$," Journal of Fluid Mechanics, vol. 187, pp. 61-98, 1988. 